\title{
Schur quadratic concavity of the elliptic Neuman mean and its application
}

\author{
Yu-Ming Chu ${ }^{1 *}$, Yan Zhang ${ }^{2}$ and Song-Liang Qiu ${ }^{2}$
}

"Correspondence:

chuyuming2005@126.com

${ }^{1}$ School of Mathematics and

Computation Science, Hunan City

University, Yiyang, 413000, China

Full list of author information is

available at the end of the article

\begin{abstract}
For $x, y>0$ and $k \in[0,1]$, we prove that the elliptic Neuman mean $N_{k}(x, y)$ is strictly Schur quadratically concave on $(0, \infty) \times(0, \infty)$ if and only if $k \in[\sqrt{2} / 2,1]$. As an application, the bounds for elliptic Neuman mean $N_{k}(x, y)$ in terms of the quadratic mean $Q(x, y)=\sqrt{\left(x^{2}+y^{2}\right) / 2}$ are presented.
\end{abstract}

MSC: 26B25; 26E60

Keywords: elliptic Neuman mean; Schur quadratically concave; quadratic mean

\section{Introduction}

Let $(x, y) \in(0, \infty) \times(0, \infty)$ and $k \in[0,1]$. Then the elliptic Neuman mean $N_{k}(x, y)$, see [1], is defined by

$$
N_{k}(x, y)= \begin{cases}\frac{\sqrt{y^{2}-x^{2}}}{c n^{-1}(x / y, k)}, & x<y, \\ x, & x=y, \\ \frac{\sqrt{x^{2}-y^{2}}}{n c^{-1}(x / y, k)}, & y<x,\end{cases}
$$

where $c n^{-1}(x, k)=\int_{x}^{1} \frac{d u}{\sqrt{\left(1-u^{2}\right)\left(k^{\prime 2}+k^{2} u^{2}\right)}}$ and $n c^{-1}(x, k)=\int_{1}^{x} \frac{d u}{\sqrt{\left(u^{2}-1\right)\left(k^{2}+k^{\prime 2} u^{2}\right)}}$ are the inverse functions of Jacobian elliptic functions $c n$ and $n c$, see $[2,3]$, respectively, and $k^{\prime}=\sqrt{1-k^{2}}$. In particular, $c n^{-1}(0, k)=\mathcal{K}(k)=\int_{0}^{\pi / 2} \frac{d t}{\sqrt{1-k^{2} \sin ^{2} t}}$ is the well-known complete elliptic integral of the first kind.

In [1] Neuman proved that $N_{k}(x, y)$ is symmetric and homogeneous on $(0, \infty) \times(0, \infty)$, and strictly decreasing with respect to $k \in[0,1]$ for fixed $(x, y) \in(0, \infty) \times(0, \infty)$ with $x \neq y$. In this context let us note that if a mean is homogeneous, then the order of its homogeneity must be 1 ; see [4].

Let us recall the notion of Schur quadratic convexity (concavity) [5-7] for a real-valued function on $(0, \infty) \times(0, \infty)$.

A real-valued function $f:(0, \infty) \times(0, \infty) \rightarrow \mathbb{R}$ is said to be strictly Schur quadratically convex on $(0, \infty) \times(0, \infty)$ if $f\left(x_{1}, x_{2}\right)<f\left(y_{1}, y_{2}\right)$ for each pair of 2-tuples $\left(x_{1}, x_{2}\right),\left(y_{1}, y_{2}\right) \in$ $(0, \infty) \times(0, \infty)$ with $\max \left\{x_{1}, x_{2}\right\}<\max \left\{y_{1}, y_{2}\right\}$ and $x_{1}^{2}+x_{2}^{2}=y_{1}^{2}+y_{2}^{2} . f$ is said to be strictly Schur quadratically concave if $-f$ is strictly Schur quadratically convex.

The main purpose of this paper is to present the range of $k$ such that the elliptic Neuman mean $N_{k}(x, y)$ is strictly Schur quadratically concave on $(0, \infty) \times(0, \infty)$. As an application,

\section{Springer}

(c)2014 Chu et al.; licensee Springer. This is an Open Access article distributed under the terms of the Creative Commons Attribution License (http://creativecommons.org/licenses/by/2.0), which permits unrestricted use, distribution, and reproduction in any medium, provided the original work is properly cited. 
an inequality between the elliptic Neuman mean $N_{k}(x, y)$ and the quadratic mean $Q(x, y)=$ $\sqrt{\left(x^{2}+y^{2}\right) / 2}$ is also given.

\section{Two lemmas}

In order to prove our main results we need two lemmas, which we present in this section.

Lemma 2.1 (See [5, Corollary 2.1], [6, Corollary 1], [7, Corollary 1]) Suppose that $f$ : $(0, \infty) \times(0, \infty) \rightarrow(0, \infty)$ is a continuous symmetric function. If $f$ is differentiable in $(0, \infty) \times(0, \infty)$, then $f$ is strictly Schur quadratically convex on $(0, \infty) \times(0, \infty)$ if and only if

$$
(x-y)\left(y \frac{\partial f(x, y)}{\partial x}-x \frac{\partial f(x, y)}{\partial y}\right)>0
$$

for all $x, y \in(0, \infty)$ with $x \neq y$, and $f$ is strictly Schur quadratically concave on $(0, \infty) \times$ $(0, \infty)$ if and only if inequality $(2.1)$ is reversed.

Lemma 2.2 Let $t \in(0,1), k \in[0,1]$, and

$$
f_{k}(t)=c n^{-1}(t, k)-\frac{\left(1+t^{2}\right) \sqrt{1-t^{2}}}{2 t \sqrt{1-k^{2}+k^{2} t^{2}}} .
$$

Then $f_{k}(t)<0$ for all $t \in(0,1)$ if and only if $\sqrt{2} / 2 \leq k \leq 1$, and there exists $\lambda=\lambda(k) \in(0,1)$ such that $f_{k}(t)<0$ for $t \in(0, \lambda)$ and $f_{k}(t)>0$ for $t \in(\lambda, 1)$ if $k \in[0, \sqrt{2} / 2)$.

Proof We distinguish for the proof two cases.

Case $1 . k=1$. Then from (2.2) one has

$$
\begin{aligned}
& f_{1}(t)=c n^{-1}(t, 1)-\frac{\left(1+t^{2}\right) \sqrt{1-t^{2}}}{2 t^{2}} \\
& =\cosh ^{-1}\left(\frac{1}{t}\right)-\frac{\left(1+t^{2}\right) \sqrt{1-t^{2}}}{2 t^{2}} \\
& =\log \left(1+\sqrt{1-t^{2}}\right)-\log t-\frac{\left(1+t^{2}\right) \sqrt{1-t^{2}}}{2 t^{2}}, \\
& f_{1}\left(1^{-}\right)=0 \text {, } \\
& f^{\prime}(t)=\frac{\left(1-t^{2}\right)\left(2-t^{2}\right)}{2 t^{3} \sqrt{1-t^{2}}}>0
\end{aligned}
$$

for all $t \in(0,1)$. (Here and in the sequel, $f\left(t^{-}\right)$and $f\left(t^{+}\right)$denote, respectively, the left and right limit of $f$ at $t$.)

From (2.3) and (2.4) we clearly see that $f_{1}(t)<0$ for all $t \in(0,1)$.

Case $2.0 \leq k<1$. Then $(2.2)$ leads to

$$
\begin{aligned}
& f_{k}\left(0^{+}\right)=-\infty, \\
& f_{k}\left(1^{-}\right)=0,
\end{aligned}
$$




$$
\begin{aligned}
f_{k}^{\prime}(t) & =-\frac{1}{\sqrt{\left(1-t^{2}\right)\left(1-k^{2}+k^{2} t^{2}\right)}}-\frac{-k^{2} t^{6}+\left(3 k^{2}-2\right) t^{4}+\left(1-3 k^{2}\right) t^{2}+k^{2}-1}{2 t^{2} \sqrt{1-t^{2}}\left(1-k^{2}+k^{2} t^{2}\right)^{3 / 2}} \\
& =-\frac{-k^{2} t^{6}+\left(5 k^{2}-2\right) t^{4}+\left(3-5 k^{2}\right) t^{2}+k^{2}-1}{2 t^{2} \sqrt{1-t^{2}}\left(1-k^{2}+k^{2} t^{2}\right)^{3 / 2}} \\
& =-\frac{\sqrt{1-t^{2}}\left[k^{2} t^{4}+\left(2-4 k^{2}\right) t^{2}+k^{2}-1\right]}{2 t^{2}\left(1-k^{2}+k^{2} t^{2}\right)^{3 / 2}} .
\end{aligned}
$$

Let

$$
g_{k}(t)=k^{2} t^{4}+\left(2-4 k^{2}\right) t^{2}+k^{2}-1
$$

Then simple computations lead to

$$
\begin{aligned}
& g_{k}(0)=k^{2}-1<0, \\
& g_{k}(1)=2\left(\frac{\sqrt{2}}{2}-k\right)\left(\frac{\sqrt{2}}{2}+k\right), \\
& g_{k}^{\prime}(t)=4 k^{2} t^{3}+4\left(1-2 k^{2}\right) t, \\
& g_{k}^{\prime}(0)=0, \\
& g_{k}^{\prime}(1)=4(1-k)(1+k)>0, \\
& g_{k}^{\prime \prime}(t)=12 k^{2} t^{2}+4\left(1-2 k^{2}\right), \\
& g_{k}^{\prime \prime}(0)=8\left(\frac{\sqrt{2}}{2}-k\right)\left(\frac{\sqrt{2}}{2}+k\right), \\
& g_{k}^{\prime \prime}(1)=4\left(1+k^{2}\right)>0 .
\end{aligned}
$$

We distinguish for the proof three subcases.

Subcase 2.1. $k=\sqrt{2} / 2$. Then (2.7) leads to the conclusion that

$$
f_{\sqrt{2} / 2}^{\prime}(t)=\frac{\sqrt{2} \sqrt{1-t^{2}}\left(1-t^{4}\right)}{2 t^{2}\left(1+t^{2}\right)^{3 / 2}}>0
$$

for all $t \in(0,1)$.

Therefore, $f_{\sqrt{2} / 2}(t)<0$ for all $t \in(0,1)$ follows from (2.6) and (2.17).

Subcase 2.2. $\sqrt{2} / 2<k<1$. Then (2.10) and (2.15) lead to

$$
\begin{aligned}
& g_{k}(1)<0, \\
& g_{k}^{\prime \prime}(0)<0 .
\end{aligned}
$$

It follows from (2.14) that $g_{k}^{\prime \prime}$ is strictly increasing on $(0,1)$, then (2.16) and (2.19) lead to the conclusion that there exists $\lambda_{0} \in(0,1)$ such that $g_{k}^{\prime}$ is strictly decreasing on $\left(0, \lambda_{0}\right]$ and strictly increasing on $\left[\lambda_{0}, 1\right)$.

From (2.12) and (2.13) together with the piecewise monotonicity of $g_{k}^{\prime}$ we clearly see that there exists $\lambda_{1} \in\left(\lambda_{0}, 1\right)$ such that $g_{k}$ is strictly decreasing on $\left(0, \lambda_{1}\right]$ and strictly increasing 
on $\left[\lambda_{1}, 1\right)$. Then $(2.9)$ and $(2.18)$ lead to the conclusion that

$$
g_{k}(t)<0
$$

for all $t \in(0,1)$.

It follows from (2.7) and (2.8) together with $(2.20)$ that $f_{k}$ is strictly increasing on $(0,1)$. Therefore, $f_{k}(t)<0$ for all $t \in(0,1)$ follows easily from $(2.6)$ and the monotonicity of $f_{k}$.

Subcase 2.3. $0 \leq k<\sqrt{2} / 2$. Then from (2.10) and (2.11) we know that $g_{k}$ is strictly increasing on $(0,1)$ and

$$
g_{k}(1)>0
$$

It follows from (2.9) and (2.21) together with the monotonicity of $g_{k}$ that there exists $\mu_{0} \in(0,1)$ such that $g_{k}(t)>0$ for $t \in\left(0, \mu_{0}\right)$ and $g_{k}(t)<0$ for $t \in\left(\mu_{0}, 1\right)$. Then $(2.7)$ and (2.8) lead to the conclusion that $f_{k}$ is strictly increasing on $\left(0, \mu_{0}\right]$ and strictly decreasing on $\left[\mu_{0}, 1\right)$. Therefore, there exists $\lambda=\lambda(k) \in\left(0, \mu_{0}\right) \subset(0,1)$ such that $f_{k}(t)<0$ for $t \in(0, \lambda)$ and $f_{k}(t)>0$ for $t \in(\lambda, 1)$ follows from (2.5) and (2.6) together with the piecewise monotonicity of $f_{k}$.

\section{Main results}

Theorem 3.1 The elliptic Neuman mean $N_{k}(x, y)$ is strictly Schur quadratically concave on $(0, \infty) \times(0, \infty)$ if and only if $k \in[\sqrt{2} / 2,1]$, and $N_{k}(x, y)$ is not Schur quadratically convex on $(0, \infty) \times(0, \infty)$ if $k \in[0, \sqrt{2} / 2)$.

Proof Since $N_{k}(x, y)$ is symmetric and homogeneous of degree 1, without loss of generality, we assume that $x<y$. Let $t=x / y \in(0,1)$, then

$$
\begin{aligned}
& N_{k}(x, y)=y N_{k}(t, 1), \quad \frac{\partial t}{\partial y}=-\frac{x}{y^{2}}, \\
& \frac{\partial N_{k}(x, y)}{\partial y}=N_{k}(t, 1)-t \frac{d N_{k}(t, 1)}{d t},
\end{aligned}
$$

Note that

$$
\frac{d N_{k}(t, 1)}{d t}=-\frac{t}{\sqrt{1-t^{2}} c n^{-1}(t, k)}+\frac{1}{\left(c n^{-1}(t, k)\right)^{2} \sqrt{1-k^{2}+k^{2} t^{2}}} .
$$

It follows from (1.1) and (3.2) together with (3.3) that

$$
\begin{aligned}
(y & -x)\left(x \frac{\partial N_{k}(x, y)}{\partial y}-y \frac{\partial N_{k}(x, y)}{\partial x}\right) \\
& =x(y-x)\left[N_{k}(t, 1)-\left(t+\frac{1}{t}\right) \frac{d N_{k}(t, 1)}{d t}\right] \\
& =\frac{2 x(y-x)}{\sqrt{1-t^{2}}\left(c n^{-1}(t, k)\right)^{2}}\left(c n^{-1}(t, k)-\frac{\left(1+t^{2}\right) \sqrt{1-t^{2}}}{2 t \sqrt{1-k^{2}+k^{2} t^{2}}}\right) \\
& =\frac{2 x(y-x)}{\sqrt{1-t^{2}}\left(c n^{-1}(t, k)\right)^{2}} f_{k}(t),
\end{aligned}
$$

where $f_{k}(t)$ is defined as in Lemma 2.2.

Therefore, Theorem 3.1 follows easily from Lemmas 2.1 and 2.2 together with (3.4). 
Theorem 3.2 The elliptic Neuman mean $N_{k}(x, y)$ is strictly Schur quadratically concave (or convex, respectively) on $(0, \infty) \times(0, \infty)$ if and only if the function $N_{k}(t, 1) / Q(t, 1)$ is strictly increasing (or decreasing, respectively) in $(0,1)$, where $Q(x, y)=\sqrt{\left(x^{2}+y^{2}\right) / 2}$ is the quadratic mean of $x$ and $y$.

Proof Without loss of generality, we assume that $x<y$. Let $t=x / y \in(0,1)$, then from (3.1) and (3.2) together with (3.4) we get

$$
\begin{aligned}
\frac{d\left(N_{k}(t, 1) / Q(t, 1)\right)}{d t} & =-\frac{\sqrt{2} t}{\left(t^{2}+1\right)^{3 / 2}}\left(N_{k}(t, 1)-\left(t+\frac{1}{t}\right) \frac{d N_{k}(t, 1)}{d t}\right) \\
& =-\frac{\sqrt{2}}{y(y-x)\left(t^{2}+1\right)^{3 / 2}}(y-x)\left(x \frac{\partial N_{k}(x, y)}{\partial y}-y \frac{\partial N_{k}(x, y)}{\partial x}\right) .
\end{aligned}
$$

Therefore, Theorem 3.2 follows easily from Lemma 2.1 and (3.5).

Theorem 3.3 The inequalities

$$
Q(x, y)>N_{\sqrt{2} / 2}(x, y)
$$

and

$$
Q(x, y)<\frac{\sqrt{2} \mathcal{K}(k)}{2} N_{k}(x, y)
$$

hold for all $x, y>0$ with $x \neq y$, and $k \in[0,1]$, and $N_{\sqrt{2} / 2}(x, y)$ is the best possible lower elliptic Neuman mean bound for the quadratic mean $Q(x, y)$.

Proof Without loss of generality, we assume that $y>x>0$. Let $t=x / y \in(0,1)$ and $L_{k}(t)=$ $N_{k}(t, 1) / Q(t, 1)$. Then

$$
\begin{aligned}
& L_{k}(t)=\frac{N_{k}(x, y)}{Q(x, y)}, \\
& L_{k}(0)=\frac{\sqrt{2}}{\mathcal{K}(k)}, \\
& L_{k}(1)=1 .
\end{aligned}
$$

We distinguish for the proof two cases.

Case $1 . k \in[\sqrt{2} / 2,1]$. Then from Theorems 3.1 and 3.2 we clearly see that $L_{k}$ is strictly increasing on $(0,1)$. Then (3.8)-(3.10) lead to the conclusion that

$$
\frac{\sqrt{2}}{\mathcal{K}(k)}<\frac{N_{k}(x, y)}{Q(x, y)}<1 .
$$

In particular, for $k=\sqrt{2} / 2$ we have

$$
\frac{N_{\sqrt{2} / 2}(x, y)}{Q(x, y)}<1 .
$$

Therefore, inequalities (3.6) and (3.7) follow from (3.11) and (3.12). 
Case 2. $k \in[0, \sqrt{2} / 2)$. Then (3.4) and (3.5) together with the Subcase 2.3 in Lemma 2.2 lead to the conclusion that there exists $\lambda=\lambda(k) \in(0,1)$ such that $L_{k}^{\prime}(t)>0$ for $t \in(0, \lambda)$ and $L_{k}^{\prime}(t)<0$ for $t \in(\lambda, 1)$, hence $L_{k}$ is strictly increasing on $(0, \lambda]$ and strictly decreasing on $[\lambda, 1)$. Therefore, $N_{k}(x, y)>Q(x, y)$ for all $x, y>0$ with $x / y \in(\lambda, 1)$ follows from (3.8) and (3.10) together with the monotonicity of $L_{k}$ on $[\lambda, 1)$, and the optimality of inequality (3.6) follows.

Note that

$$
L_{k}(0)=\frac{\sqrt{2}}{\mathcal{K}(k)}<\frac{\sqrt{2}}{\mathcal{K}(0)}=\frac{2 \sqrt{2}}{\pi}=0.90031 \cdots<1 .
$$

From (3.8), (3.9), (3.13), and the piecewise monotonicity of $L_{k}$ we clearly see that

$$
\frac{N_{k}(x, y)}{Q(x, y)}=L_{k}(t)>L_{k}(0)=\frac{\sqrt{2}}{\mathcal{K}(k)} .
$$

Therefore, inequality (3.7) follows from (3.14).

\section{Competing interests}

The authors declare that they have no competing interests.

\section{Authors' contributions}

All authors contributed equally to the writing of this paper. All authors read and approved the final manuscript.

\section{Author details}

${ }^{1}$ School of Mathematics and Computation Science, Hunan City University, Yiyang, 413000, China. ${ }^{2}$ School of Science,

Zhejiang Sci-Tech University, Hangzhou, 310018, China.

\section{Acknowledgements}

This research was supported by the Natural Science Foundation of China under Grants 11171307 and 61374086, and the Natural Science Foundation of Zhejiang Province under Grant LY13A010004.

Received: 13 July 2014 Accepted: 8 October 2014 Published: 16 Oct 2014

\section{References}

1. Neuman, E: On one-parameter family of bivariate means. Aequ. Math. 83(1-2), 191-197 (2012)

2. Carlson, BC: Special Functions of Applied Mathematics. Academic Press, New York (1977)

3. Olver, FWJ, Lozier, DW, Boisvert, RF, Clark, CW (eds.): NIST Handbook of Mathematical Functions. Cambridge University Press, Cambridge (2010)

4. Matkowski, J: Convex functions with respect to a mean and a characterization of quasi-arithmetic means. Real Anal. Exch. 29(1), 229-246 (2003/2004)

5. Yang, Z-H: Schur power convexity of Gini means. Bull. Korean Math. Soc. 50(2), 485-498 (2013)

6. Yang, Z-H: Schur power convexity of the Daróczy means. Math. Inequal. Appl. 16(3), 751-762 (2013)

7. Yang, Z-H: Schur power convexity of Stolarsky means. Publ. Math. (Debr.) 80(1-2), 43-66 (2012) 